\title{
Involvement of (pro)renin receptor in the glomerular filtration barrier
}

\author{
Atsuhiro Ichihara - Mariyo Sakoda • \\ Asako Kurauchi-Mito • Yuki Kaneshiro • Hiroshi Itoh
}

Received: 11 January 2008 /Revised: 8 February 2008 /Accepted: 11 February 2008 /Published online: 12 March 2008

(C) The Author(s) 2008

\begin{abstract}
Pro)renin receptor-bound prorenin not only causes the generation of angiotensin II via the nonproteolytic activation of prorenin, it also activates the receptor's own intracellular signaling pathways independent of the generated angiotensin II. Within the kidneys, the (pro)renin receptor is not only present in the glomerular mesangium, it is also abundant in podocytes, which play an important role in the maintenance of the glomerular filtration barrier. Recent in vivo studies have demonstrated that the overexpression of the (pro)renin receptor to a degree similar to that observed in hypertensive rat kidneys leads to slowly progressive nephropathy with proteinuria. In addition, the handle region peptide, which acts as a decoy peptide and competitively inhibits the binding of prorenin to the receptor, is more beneficial than an angiotensin-converting enzyme inhibitor with regard to alleviating proteinuria and glomerulosclerosis in experimental animal models of diabetes and essential hypertension. Thus, the (pro)renin receptor may be upregulated in podocytes under hypertensive conditions and may contribute to the breakdown of the glomerular filtration barrier.
\end{abstract}

Keywords Angiotensin.

Mitogen-activated protein kinases .

Nonproteolytic activation $\cdot$ Podocytes $\cdot$ Prorenin

A. Ichihara $(\bowtie) \cdot$ M. Sakoda $\cdot$ A. Kurauchi-Mito $•$ Y. Kaneshiro $\cdot$

H. Itoh

Departments of Anti-Aging Medicine and Endocrinology and Internal Medicine, Keio University School of Medicine,

35 Shinanomachi, Shinjuku-ku,

Tokyo 160-8582, Japan

e-mail: atzichi@sc.itc.keio.ac.jp

\section{Introduction}

When the (pro)renin receptor binds to the "handle" region of inactive prorenin, the receptor-bound prorenin gains its enzyme activity (ability to generate angiotensin I) without the proteolytic cleavage of the prosegment of prorenin in COS-7 cells $[1,2]$, presumably as a result of a conformational change. On the other hand, receptor-bound prorenin also triggers its own intracellular signaling pathways independent of the generated angiotensin II. Studies have shown that the stimulation of the (pro)renin receptor by renin/prorenin activates tyrosine phosphorylation leading to the activation of extracellular signal-related protein kinases (ERK) [3] and upregulates transforming growth factor- $\beta 1$ (TGF- $\beta 1$ ) and matrix proteins without involving angiotensin II generation in human and rat mesangial cells [4]. In this review, we focus on the latest progress in elucidating the localization, regulation, and pathophysiological roles of the (pro)renin receptor in the kidneys.

\section{Localization in the kidney}

Studies have demonstrated that the (pro)renin receptor protein and messenger ribonucleic acid (mRNA) are expressed in the mesangium cells of human kidneys [3, 4]. However, double immunohistochemical analyses using a polyclonal antirat (pro)renin receptor antibody demonstrated that the (pro)renin receptor was colocalized with a podocyte marker, podocalyxin, but not with a mesangium marker, Thy1.1, in rat kidneys [5] and electron microscopic analyses appeared to indicate the predominant presence of the rat (pro)renin receptor in podocytes, excluding the foot processes, and its absence in mesangial cells [5]. In addition, we recently detected (pro)renin receptor mRNA 
in cultured human podocytes (unpublished data). Within the glomerulus, podocytes play an important role in the maintenance of the glomerular filtration barrier and podocyte injury leads to proteinuria and initiates glomerulosclerosis resulting in the progressive loss of renal function [6]. Therefore, (pro) renin receptor in the podocytes may contribute to proteinuria and renal injury through an angiotensin-II-dependent pathway, an angiotensin-II-independent pathway, or both pathways in chronic kidney diseases.

\section{Possible upregulation of (pro)renin receptor expression under hypertensive conditions}

In the kidneys of young hypertensive stroke-prone spontaneously hypertensive rats (SHRsp), an increase by two- or threefold in the mRNA expression of the (pro)renin receptor has been observed [7]. However, the kidneys of older hypertensive SHRsp did not show an elevation in (pro)renin receptor mRNA expression. Because the binding of renin to the (pro)renin receptor lowers the (pro)renin receptor mRNA level through a promyelocytic zinc-fingerprotein-mediated negative feedback mechanism [8], a further increase in the plasma renin levels in old hypertensive SHRsp might inhibit the increase in (pro)renin receptor mRNA levels through a negative feedback loop. More recently, enhanced mRNA expression of the (pro)renin receptor was also observed in the clipped kidneys of Goldblatt hypertensive rats [9]. These results seem to provide evidence of the importance of hypertensive conditions in the regulation of (pro)renin receptor expression in the kidneys.

\section{Effects of (pro)renin receptor overexpression on the kidney}

Recent studies in transgenic rats overexpressing the human (pro)renin receptor gene nonspecifically by three to seven times demonstrated that glomerulosclerosis with proteinuria developed at 5-6months of age even in the absence of an elevation in blood pressure [10]. In the kidneys of 5- to 6month-old transgenic rats, mitogen-activated protein kinase (s) (MAPK(s)) were activated without recognizable tyrosine phosphorylation of the epidermal growth factor receptor and the expression of TGF- $\beta 1$ was enhanced. The in vivo administration of angiotensin-converting enzyme (ACE) inhibitor did not inhibit the development of glomerulosclerosis, proteinuria, MAPK activation, or TGF- $\beta 1$ expression in the kidneys despite a significant decrease in the renal angiotensin II level. As shown in Fig. 1, recombinant rat prorenin stimulated MAPK activation in human-receptorexpressed cultured cells but human receptor was unable to evoke the enzyme activity of rat prorenin. Thus, the over- expression of the human (pro)renin receptor elicits slowly progressive nephropathy via angiotensin-II-independent MAPK activation but not through the stimulation of angiotensin II generation. However, hypertension developed at 7 months of age in transgenic rats overexpressing the human (pro)renin receptor gene exclusively in smooth muscle cells [11]. Because young transgenic rats of this strain show an enhanced expression of macula densa cyclooxygenase- 2 that suppresses the tubuloglomerular feedback system and contributes to the inhibition of hypertension development [12], hypertension may not yet have developed at 6months of age or earlier. Therefore, we can interpret these observations as indicating that the hypertension may have occurred as a result of the nephropathy. However, further studies are needed to elucidate the involvement of the (pro)renin receptor in the development of hypertension.

\section{Receptor-bound prorenin in diabetic kidneys}

Recombinant prorenin binds to recombinant (pro)renin receptor expressed compulsorily on the cell surfaces of COS-7 cells and prorenin bound to the receptor on the cell surfaces exerts renin activity without undergoing any change in its molecular weight. However, synthetic peptides containing the amino acid sequence corresponding to the "handle" region of the prorenin prosegment competitively inhibit prorenin from binding to cell membrane receptors [13]. In the absence of experimental data showing the blockade of renin signaling by the handle region peptide, we believe that the possible inhibition of receptor signals and angiotensin II generation by the handle region peptide should be interpreted as indicating the blockade of prorenin binding.

Diabetic animals or patients have lower renin levels and higher prorenin levels than normal healthy controls [14, 15]. The handle region peptide was administered to rats with streptozotosin-induced type I diabetes and the tissue angiotensin I and II levels in their kidneys, and the development of nephropathy were followed [16]. The administration of the handle region peptide for 6months significantly inhibited the increase in renal angiotensin II levels and the development of proteinuria and glomerulosclerosis, suggesting that the nonproteolytic activation of prorenin bound to the (pro)renin receptor plays an important role in the development of nephropathy. Although renal mRNA expression of the (pro)renin receptor was similar in the control and diabetic rats [17], nonproteolytically activated prorenin increased in the kidneys of the diabetic rats, and this increase was inhibited by the handle region peptide. In addition, the renal total renin content remained unchanged during the treatment with the handle region peptide. These results suggested that the handle region peptide inhibits the conformational change in 
Fig. 1 Both rat and human prorenin stimulate human (pro)renin receptor $(h(P) R R)$ dependent intracellular signals, but the $h(P) R R$ activates human prorenin but does not activate rat prorenin

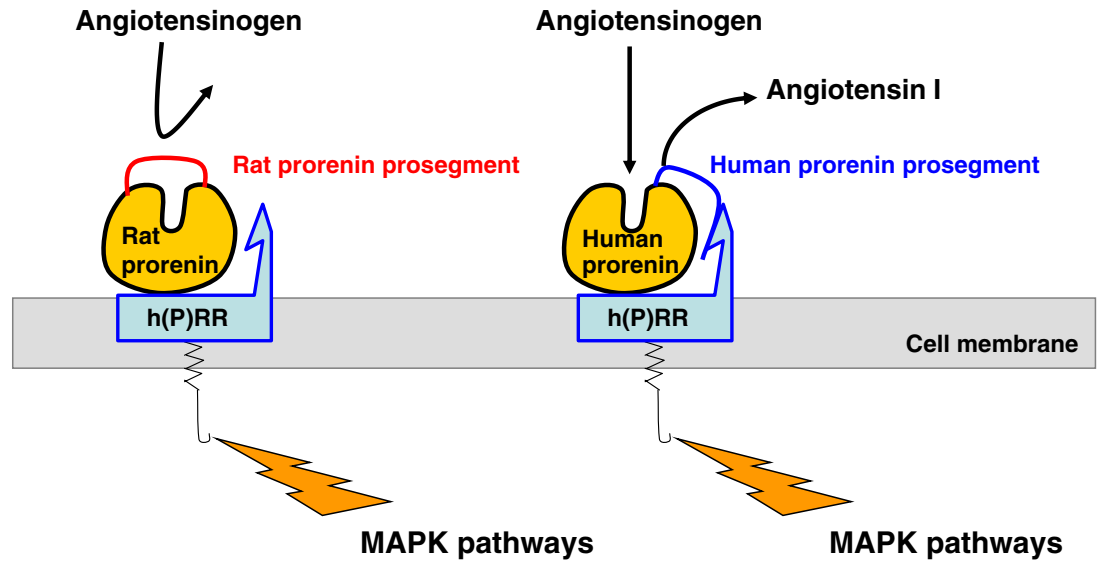

prorenin that leads to prorenin's enzymatic activation but does not alter the prorenin levels. Because the mRNA expression of cathepsin $\mathrm{B}$, which processes the conversion of prorenin to renin, simultaneously decreased in the kidneys of the diabetic rats, prorenin may have accumulated in their kidneys. Also, the amount of prorenin released to the circulatory system may be elevated in diabetes leading to an elevation in receptorbound prorenin as a result of the increased prorenin levels with no change in (pro)renin receptor expression.

To investigate the contribution of angiotensin-II-independent, (pro)renin-receptor-dependent signal pathways to diabetic end-organ damage, the therapeutic effects of infusion were compared with the effect of the deletion of angiotensin II type 1a receptor (ATKO) and ACE inhibition on streptozotosininduced type I diabetes [18]. Deletion of the angiotensin II type 1 gene attenuated the development of glomerulosclerosis and proteinuria but did not prevent these developments. ACE inhibitor also failed to prevent diabetic nephropathy despite the normalization of renal angiotensin II. On the other hand, the handle region peptide almost completely prevented the development of these conditions despite the absence of an increase in renal angiotensin levels. Of note, MAPKs were markedly activated in diabetic ATKO animals and, while the handle region peptide almost completely inhibited the activation, ACE inhibition failed to even ameliorate it. These results indicate that inhibition of the angiotensin-II-mediated pathway had a little therapeutic effect on nephropathy in the diabetic rats, whereas the blockade of receptor-bound prorenin seemed to suppress the two major pathways for diabetic nephropathy: excessive production of angiotensin II in the kidneys and renal MAPK activation. In this experiment, the renal angiotensin II levels of diabetic mice treated with the handle region peptide were similar to those of untreated wild-type mice. Because the handle region peptide did not seem to inhibit the binding of renin to the (pro)renin receptor, the physiological production of renal angiotensin II induced by renin or receptor-bound renin was probably unaffected by the handle-region-peptide-induced inhibition of receptorbound prorenin. In addition, the handle region peptide did not influence the plasma levels of renin and prorenin (receptor-unbound free renin and receptor-unbound free prorenin) or blood pressure. Thus, the handle region peptide also had no effect on the physiology of the circulating reninangiotensin system.

We recently found that the handle region peptide also leads to the regression of nephropathy in rats with streptozotosin-induced type I diabetes [17]. Diabetes was induced by streptozotosin injection in 4-week-old rats. Increased urinary protein excretion and glomerulosclerosis were observed 12 weeks later and the administration of the handle region peptide or vehicle was started. While urinary protein excretion and glomerulosclerosis had progressed in vehicle-treated 28-week-old diabetic rats, treatment with the handle region peptide significantly reduced the proteinuria and glomerulosclerosis from the levels observed at the start of treatment. This reversal of the glomerulosclerosis that had already developed in the diabetic rats suggested that receptor-bound prorenin contributes not only to the onset of nephropathy but also to its progression.

The increased plasma prorenin level might be supplied by the kidneys in which high glucose levels might inhibit the processing of prorenin to renin as described above. In addition, extrarenal tissues might also be sources of the increased prorenin level under high glucose conditions because plasma prorenin is detectable even after a bilateral nephrectomy [15]. Thus, high glucose levels may contribute to an increase in the plasma prorenin level via both renal and extrarenal mechanisms, and the elevated plasma prorenin level might trigger an increase in receptor-bound prorenin leading to nephropathy. These mechanisms may explain why the handle region peptide inhibited the development and progression of nephropathy in animal models of diabetes without affecting the high blood glucose level. Further study is needed to clarify these points. 


\section{Receptor-bound prorenin in hypertensive kidneys}

SHRsp rats have high plasma levels of prorenin and renin and are used as a model of primary hypertension [19]. In addition to the increased mRNA expression of the (pro) renin receptor as described above, elevated angiotensin I and II levels and glomerulosclerosis with proteinuria have been observed in the kidneys of young hypertensive SHRsp; administration of the handle region peptide significantly suppressed these changes without affecting the development of hypertension [7]. In addition, profibrotic changes in morphology were observed in the clipped kidneys of Goldblatt hypertensive rats with enhanced mRNA expression of the (pro)renin receptor [9]. Therefore, the increased expression of the (pro)renin receptor may play an important role in the development of renal tissues showing characteristic signs of hypertensive damage. Because recent studies have demonstrated the presence of angiotensin-II-independent, (pro)renin-receptor-dependent MAPK pathways in mesangial cells [20] and vascular smooth muscle cells [21], the beneficial effects of the handle region peptide may be mediated by both of these two major pathways that are stimulated by receptor-bound prorenin. Thus, receptor-bound prorenin also contributes to the development of hypertensive end-organ damage. This was recently supported, in part, by Susic et al., who reported that the handle region peptide significantly reduced the left ventricular mass even though the myocardial collagen content remained unchanged [22].

\section{Possible inhibition of receptor-bound prorenin by high} levels of renin

Figure 2 shows the binding of renin and prorenin to the (pro)renin receptor. Because both renin and prorenin bind to the (pro)renin receptor [3], increased renin levels may increase the amount of renin and decrease the amount of prorenin that binds to the receptor. A recent review reported that the handle region peptide offered no benefit to renintransgenic models or to a model with renovascular hypertension [23]. Because the ratio of renin to prorenin must be higher in these models than in control models, renin - but not prorenin - that has bound to the receptor must contribute to the pathogenesis that occurs in these models. Because no data showed the blockade of renin signaling by the handle region peptide, the handle region peptide did not benefit the renin-transgenic models or the model with renovascular hypertension [23]. In contrast, the handle region peptide greatly benefited diabetic animals [16-18].

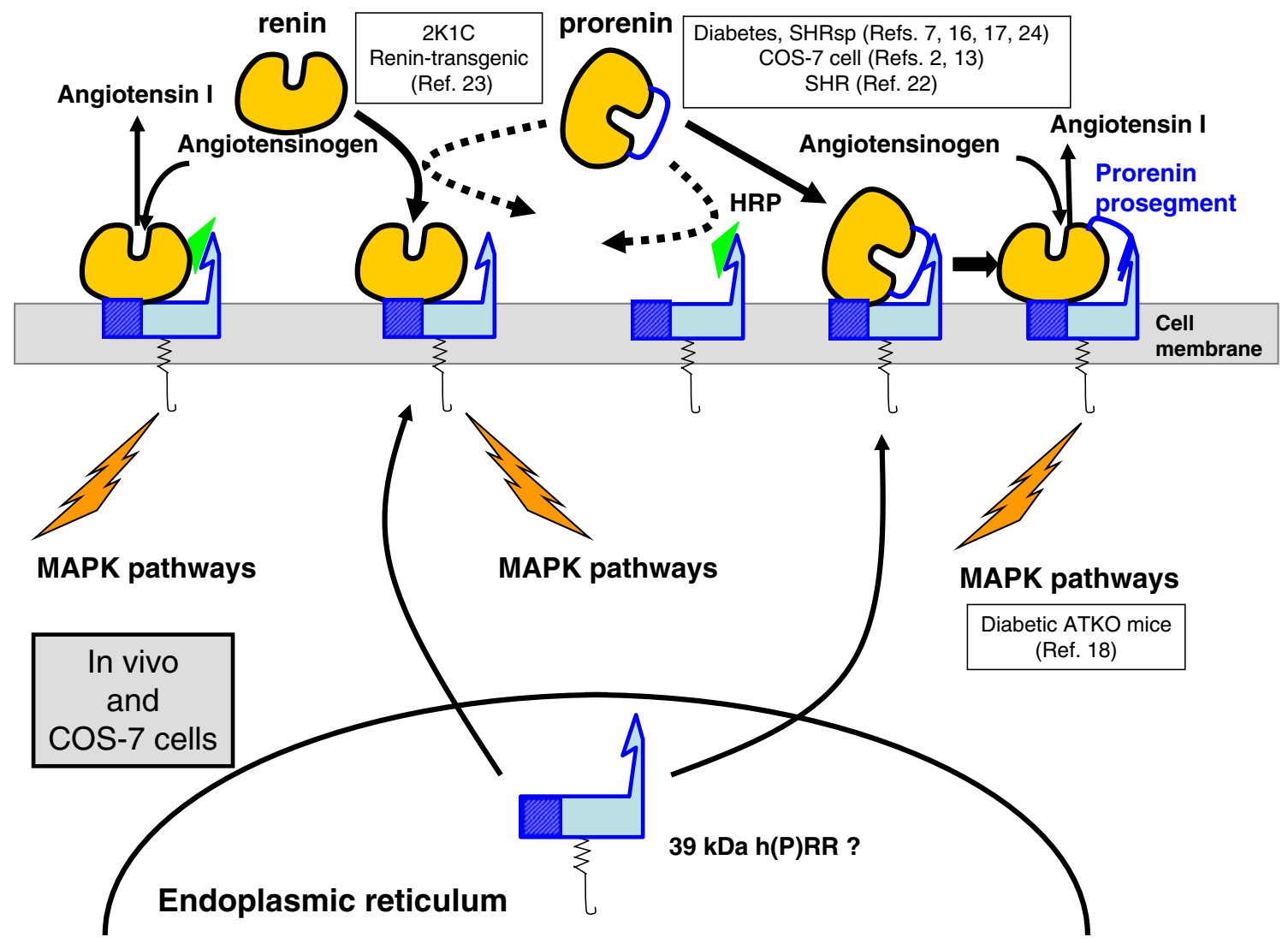

Fig. 2 Renin and prorenin competitively bind to the (pro)renin receptor in vivo and to the (pro)renin receptor expressed on the cell surfaces of COS-7 cells 
Because diabetic animals are known to have a low ratio of renin to prorenin [15], they have increased levels of prorenin bound to the receptor and decreased levels of renin bound to the receptor. Thus, the handle region peptide was thought to benefit in vivo diabetic animals by reducing the amount of prorenin that bound to the receptor. In addition, the handle region peptide had a partial benefit on SHRsp [7, 24] and SHR [22] animals fed a high-salt diet. This benefit was reduced by changing from a high-salt diet to a low-salt diet (unpublished preliminary data), suggesting that dietary salt might alter the levels of prorenin bound to the receptor by regulating the renin levels. Thus, the efficiency of the handle region peptide may depend on the ratio of renin to prorenin.

\section{(Pro)renin receptors in cultured cells}

Using vascular smooth muscle cells (VSMC) harvested from human-(pro)renin-receptor-transgenic rats, Batenburg et al. recently demonstrated that prorenin binds to the (pro) renin receptor and that receptor-bound prorenin exerts a catalytic activity but that renin does not bind to the receptor or increase its own catalytic activity [25], consistent with the results of a previous study using human VSMC [21].
The structural difference between renin and prorenin arises from 43 amino acids in the prorenin prosegment and prorenin acquires its catalytic activity without changing its molecular weight when a specific protein binds to the "handle" region of the prorenin prosegment [1]. Nevertheless, the handle region decoy peptide, which inhibited the binding of prorenin to the receptor in a test tube and to receptors expressed on the cell surfaces of COS-7 cells [13], failed to inhibit prorenin's acquisition of catalytic activity by binding to the receptor [25]. A recent study using a human cultured cell line clearly demonstrated that the $39-\mathrm{kDa}$ full-length (pro)renin receptor was predominantly present in the intracellular endoplasmic reticulum [8]. Because both renin and prorenin can permeate the cell membrane [8] but the handle region peptide cannot, the handle region peptide might be incapable of inhibiting intracellular binding between the (pro)renin receptor and its ligands (Fig. 3).

Although (pro)renin receptors of VSMC harvested from human-(pro)renin-receptor-transgenic rats were not bound to renin [25], the same authors also showed that both renin and prorenin bind to the (pro)renin receptor and stimulate receptor-dependent ERK pathways in U937 monocytes [26]. In addition, a renin-stimulated, (pro)renin-receptordependent ERK pathway was observed in rat and human

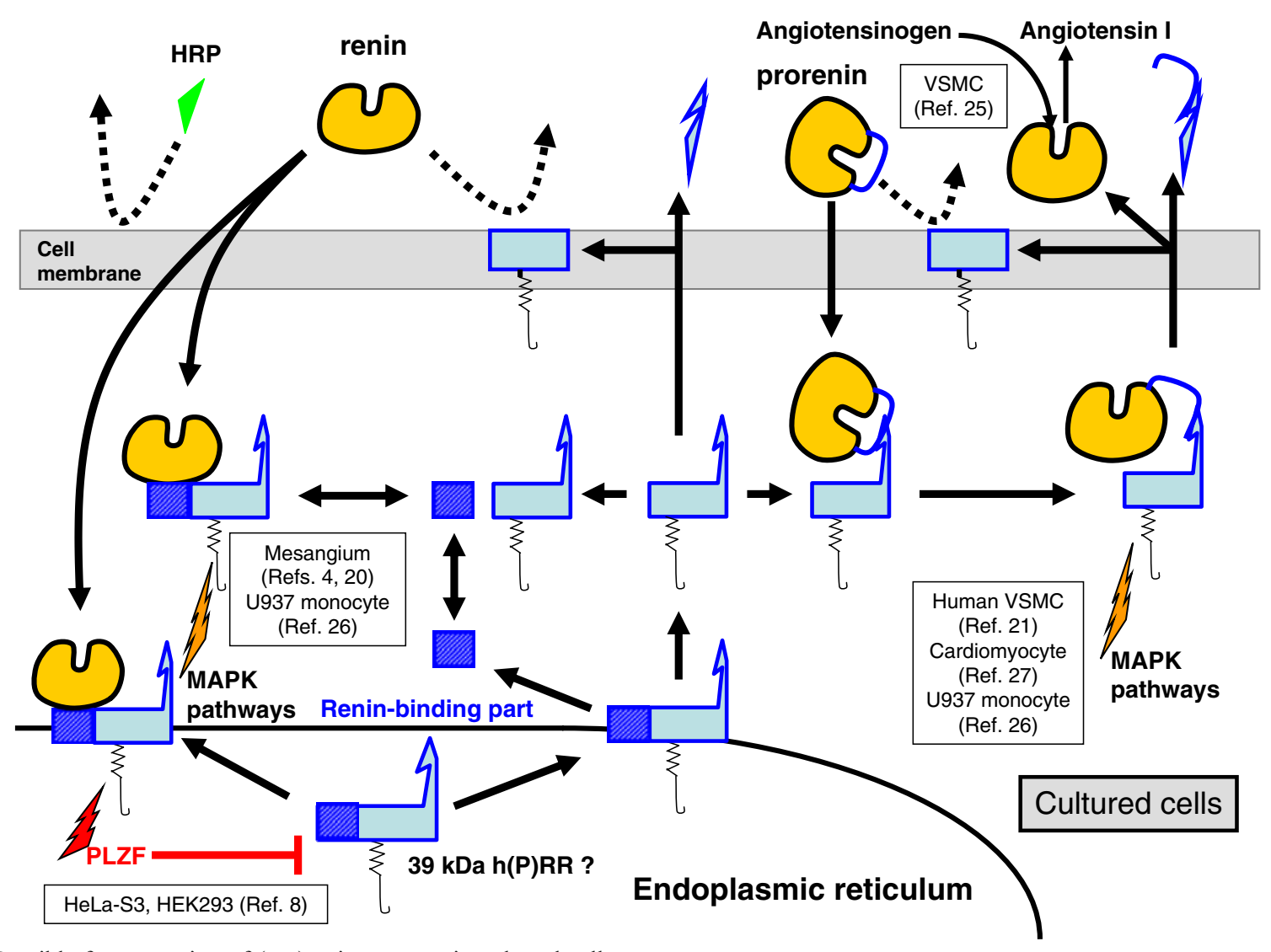

Fig. 3 Possible fragmentation of (pro)renin receptor in cultured cells 
mesangial cells $[4,20]$, whereas the stimulation of the receptor by prorenin in cultured cardiomyocytes resulted in the activation of p-38 MAPK, but not ERK [27], and renin stimulation caused the interaction between the receptor and the transcription factor promyelocytic zinc finger protein in HeLa-S3 cells and HEK293 cells [8]. These results suggest that the ability to bind renin and prorenin and the intracellular pathways involving these proteins may be influenced by the species and condition of the cultured cells. In addition, the presence of (pro)renin receptors with molecular weights smaller than the $39 \mathrm{kDa}$ of the full-length receptor in cultured cells has been reported in abstracts presented at several meetings. Based on the results of all these studies regarding the (pro)renin receptor in cultured cells, we propose the possible fragmentation of the (pro) renin receptor (Fig. 3). Namely, when the 39-kDa fulllength (pro)renin receptor in the endoplasmic reticulum is released to the cytoplasm, it may divide into a reninbinding part and a remnant part. In the cytoplasm of VSMC and cardiomyocytes, the remaining smaller (pro)renin receptor without the renin-binding part would be capable of binding prorenin but would be incapable of binding renin. However, in the cytoplasm of the mesangium and monocytes, the smaller receptor might readhere to the renin-binding part and resume its ability to bind renin. When the smaller receptor in the cytoplasm reaches the cell membrane, it might further divide into the prorenin-binding part, recognizing the "handle" region, and a remnant part. The prorenin-binding part would be released to the extracellular area and the smallest remnant receptor would remain at the cell membrane. Because the smallest receptor does not contain either the renin-binding part or the prorenin-binding part, it would no longer be capable of binding renin or prorenin. When the prorenin bound to the smaller receptor also reaches the cell membrane, it would divide into three parts: the prorenin prosegment adhered to the prorenin-binding part, renin, and the remaining smallest receptor. Because the former two proteins are released to the extracellular area, angiotensin I generation from external angiotensinogen would occur there. Thus, under cultured cell conditions, the (pro)renin receptor may be fragmented depending on the cell species. Further studies are needed to verify the possible fragmentation of the (pro) renin receptor.

\section{Conclusion}

In the glomerulus of the kidneys, the (pro)renin receptor is not only present in the mesangium but is also abundant in the podocytes and its expression was increased by two- to threefold in the kidneys of some animal models of hypertension. A transgene-induced overexpression of human (pro)renin receptor in rats resulted in the development of slowly progressive nephropathy with proteinuria. In addition, the handle-region-peptide-induced inhibition of receptorbound prorenin significantly inhibited the development and progression of proteinuria in experimental diabetic and hypertensive animal models. Thus, the glomerular (pro)renin receptor appears to play an important role in the regulation of the glomerular filtration barrier.

Acknowledgements This work was supported in part by grants from the Ministry of Education, Culture, Sports, Science and Technology of Japan (16790474 and 17390249), from the Takeda Science Foundation, and from the Japan Diabetes Foundation. We thank Ms. Miki Chika for her excellent technical assistance.

Open Access This article is distributed under the terms of the Creative Commons Attribution Noncommercial License which permits any noncommercial use, distribution, and reproduction in any medium, provided the original author(s) and source are credited.

\section{References}

1. Suzuki F, Hayakawa M, Nakagawa T, Nasir UM, Ebihara A, Iwasawa A, Ishida Y, Nakamura Y, Murakami K (2003) Human prorenin has "gate and handle" regions for its non-proteolytic activation. J Biol Chem 278:22217-22222

2. Nabi AHMN, Kageshima A, Uddin M, Nakagawa T, Park E, Suzuki F (2006) Binding properties of rat prorenin and renin to the recombinant rat renin/prorenin receptor prepared by a baculovirus expression system. Int J Mol Med 18:483-488

3. Nguyen G, Delarue F, Burckle C, Bouzhir L, Giller T, Sraer J-D (2002) Pivotal role of the renin/prorenin receptor in angiotensin II production and cellular responses to renin. J Clin Invest 109:1417-1427

4. Huang Y, Wongamorntham S, Kasting J, McQuillan D, Owens RT, Yu L, Noble NA, Border W (2006) Renin increases mesangial cell transforming growth factor-b1 and matrix proteins through receptor-mediated, angiotensin II-independent mechanisms. Kidney Int 69:105-113

5. Ichihara A, Kaneshiro Y, Takemitsu T, Sakoda M, Itoh H (2007) The (pro)renin receptor and the kidney. Semin Nephrol 27:524-528

6. Pavenstadt H, Kriz W, Kretzler M (2003) Cell biology of the glomerular podocyte. Physiol Rev 83:253-307

7. Ichihara A, Kaneshiro Y, Takemitsu T, Sakoda M, Nakagawa T, Nishiyama A, Kawachi H, Shimizu F, Inagami T (2006) Contribution of non-proteolytically activated prorenin in glomeruli to hypertensive renal damage. J Am Soc Nephrol 17:2495-2503

8. Schefe J, Menk M, Reinemund J, Effertz K, Hobbs R, Pandolfi P, Ruiz P, Unger T, Funke-Kaiser H (2006) A novel signal transduction cascade involving direct physical interaction of the renin/prorenin receptor with the transcription factor promyelocytic zinc finger protein. Circ Res 99:1355-1366

9. Krebs C, Hamming I, Sadaghiani S, Steinmetz OM, MeyerSchwesinger C, Fehr S, Stahl RAK, Garrelds IM, Danser AHJ, Goor, Contrepas A, Nguyen G, Wenzel U (2007) Antihypertensive therapy upregulates renin and (pro)renin receptor in the clipped kidney of Goldblatt hypertensive rats. Kidney Int 72:725-730

10. Kaneshiro Y, Ichihara A, Sakoda M, Takemitsu T, Nabi AN, Uddin MN, Nakagawa T, Nishiyama A, Suzuki F, Inagami T, Itoh H (2007) Slowly progressive, angiotensin II-independent glomerulosclerosis in human-renin/prorenin-receptor-transgenic rats. J Am Soc Nephrol 18:1789-1795 
11. Burckle CA, Jan Danser AH, Muller DN, Garrelds IM, Gasc JM, Popova E, Plehm R, Peters J, Bader M, Nguyen G (2006) Elevated blood pressure and heart rate in human renin receptor transgenic rats. Hypertension 47:1-5

12. Kaneshiro Y, Ichihara A, Takemitsu T, Sakoda M, Suzuki F, Nakagawa T, Hayashi M, Inagami T (2006) Increased expression of cyclooxygenase- 2 in renal cortex of human-prorenin-receptorgene transgenic rats. Kidney Int 70:641-646

13. Nabi AHMN, Uddin M, Nakagawa $T$, Iwata $H$, Ichihara $A$, Inagami T, Suzuki F (2007) Role of "handle" region of prorenin prosegment in the non-proteolytic activation of prorenin by binding to membrane anchored (pro)renin receptor. Front Biosci 12:4810-4817

14. Hayashi M, Senba S, Saito I, Kitajima W, Saruta T (1983) Changes in blood pressure, urinary kallikrein, and urinary prostaglandin E2 in rats with streptozotocin-induced diabetes. Naunyn Schmiedebergs Arch Pharmacol 322:290-294

15. Derkx F, Schalekamp M (1988) Human prorenin: pathophysiology and clinical implications. Clin Exp Hypertens A10:1213-1225

16. Ichihara A, Hayashi M, Kaneshiro Y, Suzuki F, Nakagawa T, Tada Y, Koura Y, Nishiyama A, Okada H, Uddin MN, Nabi AHMN, Ishida Y, Inagami T, Saruta T (2004) Inhibition of diabetic nephropathy by a decoy peptide corresponding to the "handle" region for non-proteolytic activation of prorenin. J Clin Invest 114:1128-1135

17. Takahashi H, Ichihara A, Kaneshiro Y, Inomata K, Sakoda M, Takemitsu T, Nishiyama A, Itoh H (2007) Regression of nephropathy developed in diabetes by (pro)renin receptor blockade. J Am Soc Nephrol 18:2054-2061

18. Ichihara A, Suzuki F, Nakagawa T, Kaneshiro Y, Takemitsu T, Sakoda M, Nabi AHMN, Nishiyama A, Sugaya T, Hayashi M, Inagami T (2006) Prorenin receptor blockade inhibits development of glomerulosclerosis in diabetic angiotensin II type 1a receptor deficient mice. J Am Soc Nephrol 17:1950-1961

19. Obata J, Nakamura T, Takano H, Naito A, Kimura H, Yoshida Y, Shimizu F, Guo DF, Inagami T (2000) Increased gene expression of components of the renin-angiotensin system in glomeruli of genetically hypertensive rats. J Hypertens 18:1247-1255

20. Huang Y, Noble N, Zhang J, Xu C, Border W (2007) Reninstimulated TGF-betal expression is regulated by a mitogenactivated protein kinase in mesangial cells. Kidney Int 72:42-52

21. Sakoda M, Ichihara A, Kaneshiro Y, Takemitsu T, Nakazato Y, Migita T, Nabi AHMN, Nakagawa T, Suzuki F, Inagami T, Itoh H (2007) (Pro)renin receptor-mediated activation of mitogen-activated protein kinases in human vascular smooth muscle cells. Hypertens Res 30:1139-1146

22. Susic D, Lippton H, Knight M, Frohlich ED (2006) Cardiovascular effects of nonproteolytic activation of prorenin. Hypertension $48: \mathrm{e} 113$

23. Luft FC (2007) Renin and its putative receptor remain enigmas. J Am Soc Nephrol 18:1989-1992

24. Ichihara A, Kaneshiro Y, Takemitsu T, Sakoda M, Suzuki F, Nakagawa T, Nishiyama A, Inagami T, Hayashi M (2006) Nonproteolytic activation of prorenin contributes to development of cardiac fibrosis in genetic hypertension. Hypertension 47:894-900

25. Batenburg WW, Krop MK, Garrelds IM, deVries R, deBruin RJA, Burckle CA, Muller DN, Bader M, Nguyen G, Danser AHJ (2007) Prorenin is the endogenous agonist of the (pro)renin receptor. Binding kinetics of renin and prorenin in rat vascular smooth muscle cells overexpressing the human (pro)renin receptor. J Hypertens 25:2441-2453

26. Feldt S, Batenburg WW, Mazak I, Maschke U, Wellner M, Kvakan H, Dechend R, Fiebeler A, Burckle C, Contrepas A, Danser AHJ, Bader M, Nguyen G, Luft FC, Muller DN (2008) Prorenin and renin-induced extracellular signal regulated kinase $1 / 2$ activation in monocytes is not blocked by aliskiren or the handle-region peptide. Hypertension 51:682-688

27. Saris J, 'tHoen P, Garrelds I, Dekkers D, denDunnen J, Lamers J, JanDanser A (2006) Prorenin induces intracellular signaling in cardiomyocytes independently of angiotensin II. Hypertension 48:564-571 